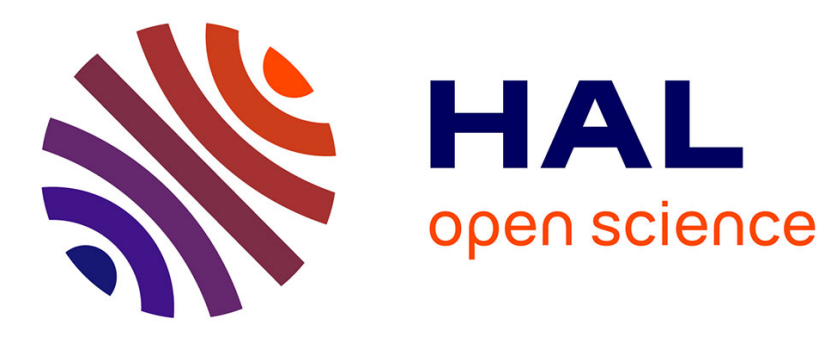

\title{
Phenomenological model for phase transformation characteristics of textured shape memory alloys
}

Dimitrios Chatziathanasiou, Yves Chemisky, Fodil Meraghni, Georges

Chatzigeorgiou, Etienne Patoor

\section{To cite this version:}

Dimitrios Chatziathanasiou, Yves Chemisky, Fodil Meraghni, Georges Chatzigeorgiou, Etienne Patoor. Phenomenological model for phase transformation characteristics of textured shape memory alloys. TMS Middle East - Mediterranean Materials Congress on Energy and Infrastructure Systems (MEMA 2015), Jan 2015, Doha, Qatar. 10.1002/9781119090427.ch21 . hal-01203603

\section{HAL Id: hal-01203603 \\ https://hal.science/hal-01203603}

Submitted on 5 Dec 2016

HAL is a multi-disciplinary open access archive for the deposit and dissemination of scientific research documents, whether they are published or not. The documents may come from teaching and research institutions in France or abroad, or from public or private research centers.
L'archive ouverte pluridisciplinaire HAL, est destinée au dépôt et à la diffusion de documents scientifiques de niveau recherche, publiés ou non, émanant des établissements d'enseignement et de recherche français ou étrangers, des laboratoires publics ou privés. 


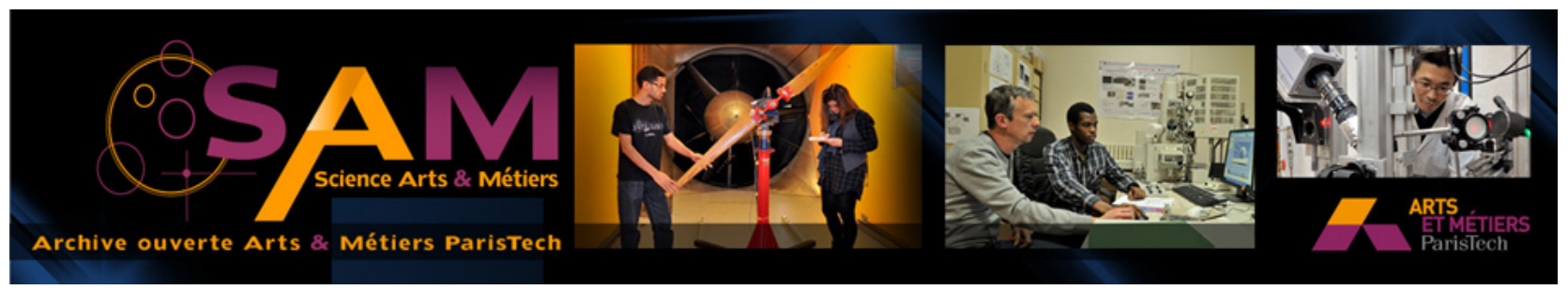

Science Arts \& Métiers (SAM)

is an open access repository that collects the work of Arts et Métiers ParisTech researchers and makes it freely available over the web where possible.

This is an author-deposited version published in: http://sam.ensam.eu Handle ID: .http://hdl.handle.net/null

\section{To cite this version :}

Dimitrios CHATZIATHANASIOU, Yves CHEMISKY, Fodil MERAGHNI, George CHATZIGEORGIOU, Etienne PATOOR - Phenomenological model for phase transformation characteristics of textured shape memory alloys - 2015 


\title{
PHENOMENOLOGICAL MODEL FOR PHASE TRANSFORMATION CHARACTERISTICS OF TEXTURED SHAPE MEMORY ALLOYS
}

\author{
Dimitris Chatziathanasiou, Yves Chemisky, Fodil Meraghni, George Chatzigeorgiou, Etienne Patoor
}

Arts et Métiers ParisTech, LEM3 UMR 7239 CNRS, 4 rue Augustin Fresnel; Metz, F-57078 cedex 01, France

Keywords: Shape memory alloys, Superelasticity ,Anisotropy, Tension-compression asymmetry, Transformation criteria, Evolution equation

\begin{abstract}
In the present study, a new transformation criterion that includes the effect of tensioncompression asymmetry and texture-induced anisotropy is proposed and combined with a thermodynamical model to describe the thermomechanical behavior of polycrystalline shape memory alloys. An altered Prager criterion has been developed, introducing a general transformation of the axes in the stress space. A convexity analysis of such criterion is included along with an identification strategy aimed at extracting the model parameters related to tension-compression asymmetry and anisotropy. These are identified from a numerical simulation of a SMA polycrystal, using a self-consistent micromechanical model previously developed by Patoor et al. (Patoor, E., Eberhardt, A., Berveiller, M., 1996. Micromechanical Modelling of Superelasticity in Shape Memory Alloys. Journal de Physique IV 6, C1 277) for several loading cases on isotropic, rolled and drawn textures. Transformation surfaces in the stress and transformation strain spaces are obtained and compared with those predicted by the micromechanical model. The good agreement obtained between the macroscopic and the microscopic polycrystalline simulations states that the proposed criterion and transformation strain evolution equation can capture phenomenologically the effects of texture on anisotropy and asymmetry in SMAs.
\end{abstract}

\section{Introduction}

Recent experimental characterization of the superelastic behavior of SMAs has shown that such materials exhibit a tension-compression asymmetry during forward transformation $[1,2]$. Indeed, the critical stress for the onset of transformation is different between tension and compression. A Mises-type criterion is thus not able to accurately predict the transformation surface in the stress space at a given temperature. It has been shown, using a micromechanical model, that the tension-compression asymmetry is linked to the loss of symmetry induced by the appearance of martensitic variants [3]. The tension-compression asymmetry is observable not only on the critical stress to trigger the martensitic transformation, but also on the stress-temperature slopes of the phase diagram and on the magnitude of transformation strains [4]. The behavior of processed SMAs also shows a strong anisotropic behavior $[5,6]$. Like other metals, the texture of 
the material and the presence of internal stresses are found to be responsible for such anisotropy. A micromechanical analysis has shown that the transformation of a textured polycrystal is strongly anisotropic [7]. Recent efforts have focused on the development of suitable criteria to take into account the anisotropic behavior of precessed SMAs. It is shown that the transformation surfaces could be well predicted but the evolution of transformation strains should be investigated even further [8].

In this work, an effort to produce a new suitable transformation criterion for loading of SMAs under a constant temperature is presented. The purpose is to introduce a criterion suitable to express both asymmetry and anisotropy and also captures multiple results that might come either from experiments or from micromechanical simulations. The second main purpose of the work is to develop a formulation that can predict the evolution of transformation strains for textured materials. The introduction of the effect of processing conditions, i.e. anisotropic transformation behavior, is of great importance, since most of the SMA structures are made out of wires or plates. The effect of drawn and rolled processing conditions have to be taken properly into account to obtain an efficient, accurate design tool.

A micromechanical model has previously been developed based on the reference work of Patoor et al [9]. The input to the micromechanical model is crystallographic orientation of the grains that forms the SMA polycrystal. The expected outcome from a polycrystal with random orientation is isotropic yield surface and inelastic strain evolution, whereas textured polycrystal would exhibit anisotropy in transformation [10, 11]. By conducting the micromechanics calculations of textured material and then calibrating the proposed equations, the aim is to come up with a consistent scheme which enables the direct connection between the processing conditions and the macroscopic effect on anisotropy that can be implemented in FEA packages for structural design.

The present paper is organized as follows: In the first part of this study, the mathematical configuration of the new criterion is presented. In the second part, three ways of achieving the transition to the space of strains are presented and discussed. The third part is dedicated to the evaluation of the new criterion using results acquired from the micromechanical model and to a discrussion about the capability of the present development to capture texture effects.

\section{New transformation criterion and evolution equation for transformation strain}

In the classic scope of thermodynamic potentials, we consider that there exists a thermoelastic region, given by the function $\Phi(\mathbf{A})$

$$
\Phi(\mathbf{A}) \leqslant 0 \quad \text { for all possible sets of } \mathbf{A} .
$$

where $\mathbf{A}$ is the set of all GTFs of the respective internal variables $\mathbf{V}$ of the material $[12,13,14,15,16]$. The transformation criterion

$$
\Phi(\mathbf{A})=0
$$

is the mathematical bound of this region. 
The principle of maximum dissipation [17] has been implemented in some successful models $[18,19]$. According to it:

$$
\dot{\mathbf{V}}=\dot{\lambda} \frac{\partial \Phi}{\partial \mathbf{A}}, \dot{\lambda}>0
$$

Viewing the transformation strain $\varepsilon^{t}$ as an internal variable, having established that the stress $\boldsymbol{\sigma}$ is its GTF [12], an evolution rule of the transformation strains is reached:

$$
\dot{\varepsilon}^{t}=\dot{\lambda} \frac{\partial \Phi}{\partial \boldsymbol{\sigma}} .
$$

Similarly, this time avoiding associativity, a transformation potential $Z(\boldsymbol{A})$ could be defined, from which $\dot{\varepsilon}^{t}$ is considered to be derived:

$$
\dot{\varepsilon}^{t}=\dot{\lambda} \frac{\partial Z}{\partial \boldsymbol{\sigma}} \text {. }
$$

The notion of the dissipation potential for the case of plasticity is well described in [16], with $\dot{\lambda}$ being the plastic multiplier.

Introduction of the altered Prager criterion

Patoor et al. [3] made use of the Prager equation to fit isotropic asymmetric results obtained from their self-consistent micromechanical model. The original form is:

$$
h(\boldsymbol{\sigma})=\sqrt{J_{2}}\left(1+b \frac{J_{3}}{J_{2}^{3 / 2}}\right)^{\frac{1}{2}}-k=0
$$

where $J_{2}$ and $J_{3}$ denote the second and third invariant of the deviatoric part of the stress tensor respectively:

$$
J_{2}=\frac{1}{2} \sigma_{i j}^{\prime} \sigma_{i j}^{\prime} \text { and } J_{3}=\frac{1}{3} \sigma_{i j}^{\prime} \sigma_{j k}^{\prime} \sigma_{k i}^{\prime}
$$

using the Einstein summation for double indices.

In this paper, the notion of the alteration of the axes of the stress space is used, as in the work of Karafillis and Boyce [20] to retain the shape of the original transformation surface, but at the same time include anisotropy. On the other hand, the expression is extended to a more general form using a power function. Thus:

$$
\hat{\Phi}_{\sigma}(\boldsymbol{\sigma})=\sqrt{J_{2}\left(\boldsymbol{\sigma}^{*}\right)}\left[1+b \frac{J_{3}\left(\boldsymbol{\sigma}^{*}\right)}{J_{2}^{3 / 2}\left(\boldsymbol{\sigma}^{*}\right)}\right]^{\frac{1}{n}}-k_{\sigma}=0
$$

where $\mathrm{n}$ is a positive real number,

$$
\boldsymbol{\sigma}^{*}=\boldsymbol{R}_{\boldsymbol{\sigma}}: \boldsymbol{\sigma}-\boldsymbol{x}_{\boldsymbol{\sigma}}
$$

$\boldsymbol{R}_{\boldsymbol{\sigma}}$ is a fourth order dimensionless tensor which only contains constants

and

$\boldsymbol{x}_{\boldsymbol{\sigma}}$ is a second order tensor with dimensions of stress.

In that way, a new space of stresses $\boldsymbol{\sigma}^{*}$ is created, one that represents a distortion of the space of real stresses and is based on the nature of the tensor $\boldsymbol{R}_{\boldsymbol{\sigma}}$, the description of which is given below: 
1. If the effects that $\boldsymbol{R}_{\boldsymbol{\sigma}}$ induces on the shear stresses are ignored and a threedimensional vector $\vec{\sigma}$ consisted of only the normal stresses is considered, then $\boldsymbol{R}_{\boldsymbol{\sigma}}$ reduces to a rotation matrix expressed as $\boldsymbol{R}$. It is found as a product of three rotation matrices:

$$
\boldsymbol{R}=\boldsymbol{R}_{z} \boldsymbol{R}_{x} \boldsymbol{R}_{p}
$$

Each of these three matrices represent a simple rotation of the space of normal stresses $\sigma_{11}-\sigma_{22}-\sigma_{33}$ and can be formed out of a single value representing the respective angle:

$\boldsymbol{R}_{\boldsymbol{z}}$ represents a rotation around the $\sigma_{33}$ axis by an angle $\theta_{z}$.

$\boldsymbol{R}_{\boldsymbol{x}}$ represents a rotation around the $\sigma_{11}$ axis by an angle $\theta_{x}$.

$\boldsymbol{R}_{\boldsymbol{p}}$ represents a rotation around the $\sigma_{11}=\sigma_{22}=\sigma_{33}$ axis by an angle $\theta_{p}$.

The result of this expression makes $\boldsymbol{R}$ a rotation matrix itself: It is orthogonal and $\operatorname{Det}(\boldsymbol{R})=1$.

2. Extending $\boldsymbol{R}$ in order to achieve a transformation of the six-dimensional space of stresses including the shears, results in the fourth-order $\boldsymbol{R}_{\boldsymbol{\sigma}}$.

Instead of extending the notion of rotation to six dimensions, the transformation of shears is expressed in a simpler way, which is simply the homogeneous anisotropic scaling of the respective axes: in the basic configuration $(7), \hat{\Phi}_{\sigma}$ is expressed by simple scaled functions of the shear stresses:

$$
\sigma_{i j}^{*}=l_{i j} \sigma_{i j} \text { for } i \neq j \text { where } l_{i j}=l_{j i}>0 .
$$

The Einstein summation is not used here.

Eventually, $\boldsymbol{R}_{\boldsymbol{\sigma}}$ introduces the operation of distortion rather of rotation.

On the other hand, $\boldsymbol{x}_{\boldsymbol{\sigma}}$ simply represents a translation of the origin of axes by the constant vector $\left\{\sigma_{o 1}, \sigma_{o 2}, 0\right\}$.

It is worth noting that the operation $\boldsymbol{R}_{\boldsymbol{\sigma}}: \boldsymbol{\sigma}$ is not equivalent to a rotation of the system coordinates, as in

$$
\sigma^{*}=R . \sigma R^{T}
$$

except if $\boldsymbol{R}$ is the fourth-order identity tensor. However in that case, the isotropic formulation is reached again.

The proposed transformation function proves to be convex when:

$$
|b|<\frac{n \sqrt{27}}{2(9-n)} \leqslant \frac{\sqrt{27}}{2} \text { for } n<4.5 .
$$

$\underline{\text { Evolution equations of transformation strain }}$

The most general form of the relation between the rates of stress and transformation strain is identical to (5), choosing a transformation potential $\hat{Z}(\boldsymbol{\sigma})$. Following a direct relation with the martensitic volume fraction $(\mathrm{MVF}) \xi[18,21]$, a more specific equation is found: 


$$
\dot{\varepsilon}^{t}=\dot{\xi} \frac{\partial \hat{Z}}{\partial \boldsymbol{\sigma}} .
$$

When $\hat{Z}(\boldsymbol{\sigma})$ is chosen to be the transformation surface, the maximum dissipation principle is satisfied [19], and:

$$
\dot{\varepsilon}^{t}=\dot{\xi} H \frac{\partial \Phi}{\partial \boldsymbol{\sigma}}
$$

In this work, it is examined how three particular evolution rules for the transformation strain behave with comparison to simiulated experimental results. The first rule is consistent with (13) and the second with (12). In addition to that, a third evolution rule will be introduced, $\dot{\varepsilon}_{\boldsymbol{r}}^{t}$, and the total of three options given will be compared.

Firstly, we define

$$
\dot{\varepsilon}_{\boldsymbol{a s}}^{t}=\dot{\xi} H \frac{\partial \hat{\Phi}_{\sigma}}{\partial \boldsymbol{\sigma}} .
$$

This formulation is consistent with the maximum dissipation principle and expresses the rule of associativity. The variable $H$ is a scale factor depending on stresses and expresses the magnitude of the rate at which $\varepsilon^{t}$ increases [22].

Secondly, we define

$$
\dot{\varepsilon}_{i s o}^{t}=\dot{\xi} H \frac{\partial \hat{\Phi}_{\sigma}^{o}}{\partial \boldsymbol{\sigma}}
$$

where

$$
\hat{\Phi}_{\sigma}^{o}(\boldsymbol{\sigma})=\sqrt{J_{2}(\boldsymbol{\sigma})}\left[1+b \frac{J_{3}(\boldsymbol{\sigma})}{J_{2}^{3 / 2}(\boldsymbol{\sigma})}\right]^{\frac{1}{n}}-k_{\sigma}=0 .
$$

This formulation comes from the consideration of $\hat{\Phi}_{\sigma}^{o}$ found in (16) as the dissipation potential $Z$ found in (5). The derivatives $\frac{\partial \hat{\Phi}_{\sigma}^{o}}{\partial \boldsymbol{\sigma}}$ and $\frac{\partial \hat{\Phi}_{\sigma}}{\partial \boldsymbol{\sigma}}$ found in (15) and (14) respectively express a tensor which is normal to the respective potentials. The first derivative is called $\boldsymbol{\Lambda}_{\boldsymbol{o}}: \boldsymbol{\Lambda}_{\boldsymbol{o}}(\boldsymbol{\sigma})=\frac{\partial \hat{\Phi}_{\sigma}^{o}(\boldsymbol{\sigma})}{\partial \boldsymbol{\sigma}}$.

On the other hand, it is found that:

$$
\frac{\partial \hat{\Phi}_{\sigma}}{\partial \boldsymbol{\sigma}}=\boldsymbol{R}_{\boldsymbol{\sigma}}: \boldsymbol{\Lambda}_{\boldsymbol{o}}\left(\boldsymbol{\sigma}^{*}\right)
$$

Finally, we define

$$
\dot{\varepsilon}_{r}^{t}=\dot{\xi} H\left(\boldsymbol{r}_{\varepsilon} \cdot \Lambda_{o} \cdot r_{\varepsilon}^{T}\right)
$$

Here, a rotation of the normal $\Lambda_{o}$ of $\dot{\varepsilon}_{2}^{t}$ is introduced, thus rotating the whole tensor that comes from the expression (15). This rotation comes from an angle $a^{\varepsilon}$ lying on the plane given by $\varepsilon_{11}^{t}+\varepsilon_{22}^{t}+\varepsilon_{33}^{t}=0$.

However, unlike $\theta_{p}$ which is considered constant as found in $\boldsymbol{R}_{\boldsymbol{p}}$, the value of $a^{\varepsilon}$ is a function of $\boldsymbol{\Lambda}_{\boldsymbol{o}}$ :

$$
a^{\varepsilon}=a_{o}^{\varepsilon}+2 \sqrt{\frac{F^{\varepsilon}}{\pi}} \operatorname{Exp}\left[-F^{\varepsilon}\left(\left(\omega-\omega_{o}^{\varepsilon}\right)^{2}+\left(c^{\varepsilon}\right)^{2}\right)\right] \sinh \left[2 F^{\varepsilon} c^{\varepsilon}\left(\omega-\omega_{o}^{\varepsilon}\right)\right] .
$$




\begin{tabular}{clc}
\hline Label of simulation & Loading conditions & Relative angle to 1-1 axis \\
\hline 1 & Uniaxial tension in 1 & $0^{\circ}$ \\
2 & Biaxial tension in 1 and 2 & $45^{\circ}$ \\
3 & Uniaxial tension in 2 & $90^{\circ}$ \\
4 & Compression in 1 - tension in 2 & $135^{\circ}$ \\
5 & Uniaxial compression in 1 & $180^{\circ}$ \\
6 & Biaxial ompression in 1 and 2 & $225^{\circ}$ \\
7 & Uniaxial compression in 2 & $270^{\circ}$ \\
8 & Tension in 1 - compression in 2 & $315^{\circ}$ \\
\hline
\end{tabular}

Table 1: Loading paths for the polycrystal specimens. The non-uniaxial cases represent equibiaxial conditions

In the latter,

$$
\omega\left(\boldsymbol{\Lambda}_{\boldsymbol{o}}: \boldsymbol{B}_{\boldsymbol{o}}^{\boldsymbol{\varepsilon}}\right)= \begin{cases}\arccos \left(\boldsymbol{\Lambda}_{\boldsymbol{o}}: \boldsymbol{B}_{\boldsymbol{o}}^{\boldsymbol{\varepsilon}}\right) ; & \Lambda_{22} \geqslant 0, \\ 2 \pi-\arccos \left(\boldsymbol{\Lambda}_{\boldsymbol{o}}: \boldsymbol{B}_{\boldsymbol{o}}^{\boldsymbol{\varepsilon}}\right) ; & \Lambda_{22}<0 .\end{cases}
$$

The rest of the variables that appear other than $\boldsymbol{\Lambda}_{\boldsymbol{o}}$ are constants. The equation (19) introduces the rotation necessary to capture the effect of anisotropy to $\dot{\varepsilon}^{t}$. It is considered that $a_{o}^{\varepsilon}=-\theta_{p}$, whereas $\boldsymbol{B}_{\varepsilon}^{o}$ takes the following form:

$$
\boldsymbol{B}_{\varepsilon}^{\boldsymbol{o}}=\left(\begin{array}{ccc}
2^{-1 / 2} & 0 & 0 \\
0 & 0 & 0 \\
0 & 0 & -2^{-1 / 2}
\end{array}\right)
$$

The choice for the tensor of reference $\boldsymbol{B}_{\boldsymbol{o}}^{\varepsilon}$ as the origin for measuring the angles is arbitrary. It represents the intersection of the $\varepsilon_{11}^{t}+\varepsilon_{22}^{t}+\varepsilon_{33}^{t}=0$ with the plane $\varepsilon_{22}^{t}=0$. The criterion $\Lambda_{22} \geqslant 0$ found in (20) is a direct result of this choice.

The variables $c^{\varepsilon}, F^{\varepsilon}, \omega_{o}^{\varepsilon}$ should be viewed as material constants and calibrate them according to experimental results. Another remark for the evolution rule introduced in $(18)$ is that it is not given as a direct derivative of a dissipation potential. A surface $\hat{Z}(\boldsymbol{\sigma})$, for which every $\dot{\varepsilon}_{r}^{t}$ is normal to, exists but its exact form is unknown.

\section{Assessment of the transformation function and evolution rules}

The numerical simulation of the constitutive response of polycrystalline SMAs are based on a micro mechanical model developed by Patoor et al. [9], implementing the selfconsistent scale transition method [23]. The simulations are carried out using the microstructural parameters of a $\mathrm{Cu}-\mathrm{Zn}-\mathrm{Al}$ alloy, taken from [23].

All the results of that model are obtained after running the simulations of loading of three polycrystal specimens containing 1000 grains each in total. These polycrystals are considered to correspond to an isotropic, rolled or drawn specimen, according to their texture. For the rolled specimen, the rolling direction was set to be along the axis 1-1. For the drawn specimen, the drawing direction was also set along the axis 1-1. The results presented hereafter correspond to simulations following eight proportional loading 
paths assuming plane stress conditions with $\sigma_{33}=\sigma_{12}=0$ in stress-controlled loading. The loading paths are presented in Table 1.

Evaluation of the proposed transformation function

The first set of simulations represent proportional loading of a non-treated polycrystal. The resulting surfaces in the stress and the strain spaces were isotropic. Their shape is found in Figures 1 and 3. The results were similar to those reported in [1] and [24].

The second set of simulations represents proportional loading of the rolled specimen. The value of $b$ was chosen at 0.65 . Two more anisotropic surfaces were calibrated for a different assumption of the value of the factor $b$, in order to demonstrate the adaptability of the transformation criterion.

The third set of simulations represents proportional loading of the drawn specimen. Because of the higher level of anisotropy, b was selected here to be a target value and was calibrated at $\mathrm{b}=0.59$.

Figures 1 and 3 show a comparison between all the results of the simulations and the criterion in terms of stresses obtained by (5), considering $n=2$. Good agreement is observed. Furthermore, it is shown in Figure 2 that using alternative values for b might result to better surfaces, in the sense that they can capture better the basic material properties, which here are the uniaxial strengths. Given an abundance of data, better calibration is induced by treating $\mathrm{b}$ as another target value. To that respect, two more loading paths are considered for the rolled specimen, labelled 9 and 10 in Figures 1 and 2. It appears that the extreme in terms of convexity value $b=0.74$ is not as accurate as the values $b=0.65$ and $b=0.3$.

The evolution of the transformation surface as a function of $\xi$ also presents a very interesting effect. The detail of the transformation surfaces corresponding to two different MVFs in Figure 3-b reveals that the sense of anisotropy may switch between directions

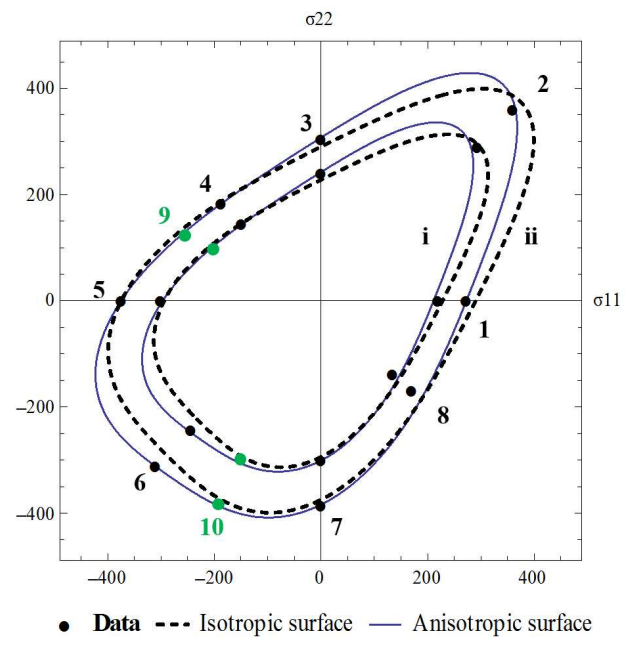

Figure 1: Evolution of transformation surface of rolled specimen for (i) $\xi=20 \%$ and (ii) $\xi=60 \%$ and comparison with isotropic surface

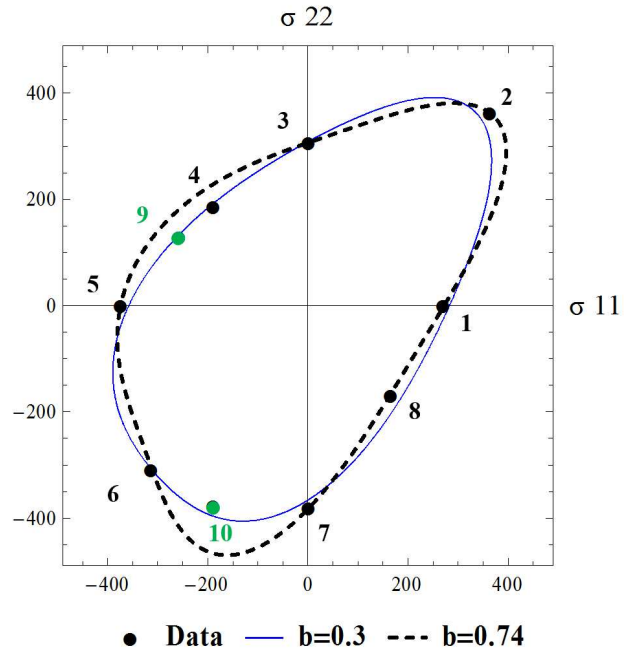

Figure 2: Comparison between two anisotropic surfaces for different values of $b$ for the rolled specimen 


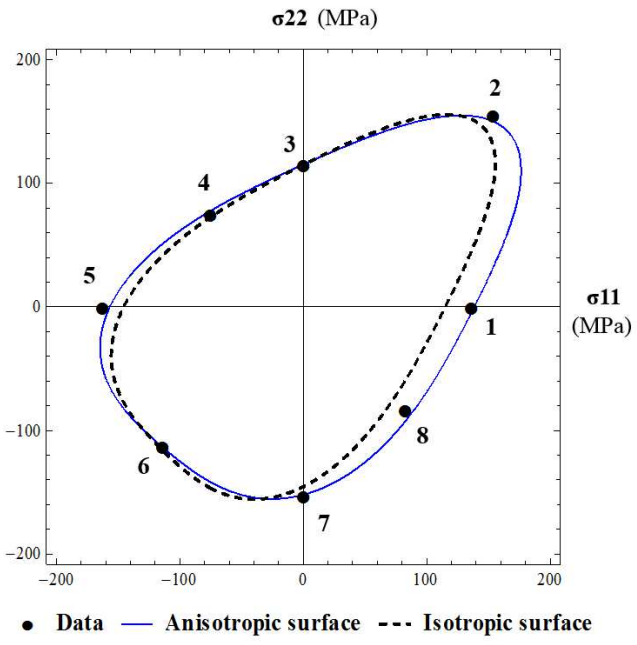

(a)

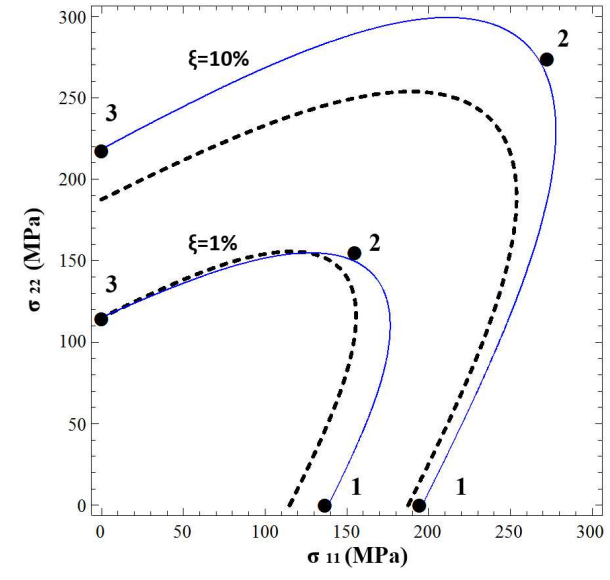

- Data — Anisotropic surface - -- Isotropic surface

(b)

Figure 3: Comparison between isotropic and anisotropic surfaces for the drawn specimen for $\xi=1 \%$ (a) and evolution of transformation surface for $\xi=1 \%$ and $\xi=10 \%$ (b).

as forward transformation progresses. Indeed, it is clear in Figure 4 that the stress which corresponds to $1 \%$ MVF for uniaxial tension in the 1-1 direction is higher than the stress for uniaxial tension in the 2-2 direction; but this is not the case when $\xi=10 \%$ : here, the stress is higher for tension along 2-2. The resulting surfaces accommodating this data are different in shape and not just in size. The size effect would be captured by a direct dependence of $\mathrm{k}$ with $\xi$, and this kind of evolution would be recognized as isotropic hardening [16]. However, in this case, more material parameters have changed between the two MVFs to capture the changing sense of anisotropy. Thus, an evolution of the material parameters is deemed necessary to capture the resulting stress-MVF curves.

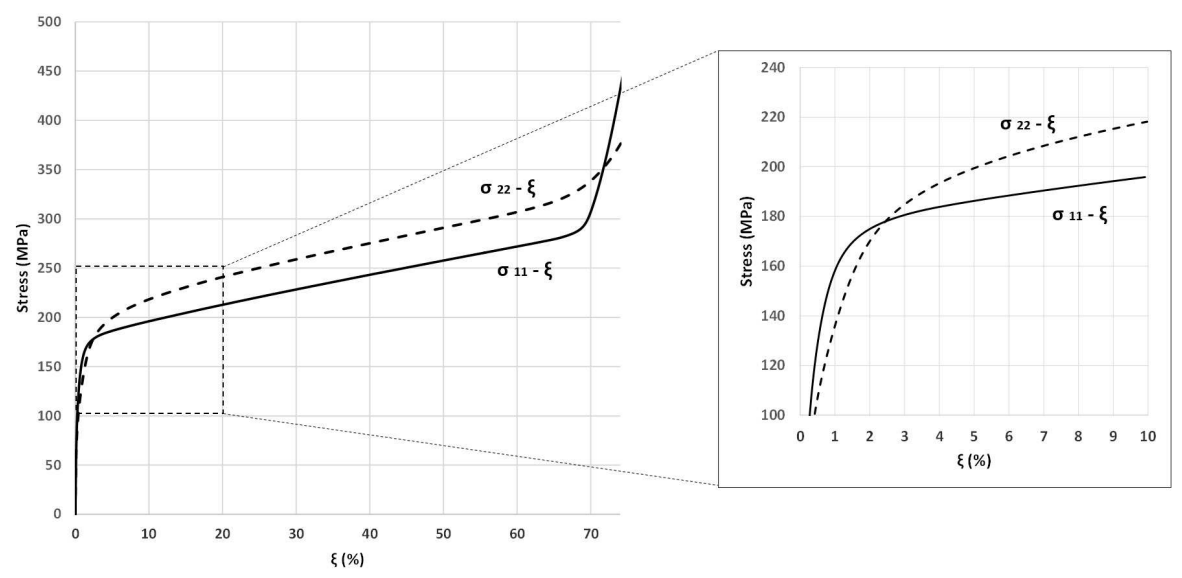

Figure 4: Stress-MVF diagram for uniaxial tension in directions 1-1 and 2-2 
Using the evolution rules (14), (15) and (18), a large number of results regarding transformation strains was gathered. These results correspond to proportional loading under various directions. For every value of $\xi$, each evolution rule results in a respective locus on which the components of $\varepsilon^{t}$ lie. In the space of normal transformation strains, the three loci are flat shapes, meaning they all lie on a plane.

Even though the two resulting loci corresponding to the to the normal to the isotropic surface evolution and the proposed evolution rule are identical, the respective evolution rules are not equivalent. While the shape on which they lie is common in the space of normal strains, the same loading direction corresponds to different positions on the shape.In Figures 5-a and 5-b, only the strains resulting from proposed criterion are accurate enough to be compared with data points. For every point on the surfaces corresponding to $\dot{\varepsilon}_{\boldsymbol{i s o}}^{t}$ and $\dot{\varepsilon}_{\boldsymbol{r}}^{t}$, it is $\varepsilon_{33}^{t}=-\varepsilon_{11}^{t}-\varepsilon_{22}^{t}$.

A notable conclusion drawn from the results of the micromechanics simulations is the strong effect of texture on the anisotropy of transformation strains. The point 7 on Figure 5-a corresponds to the response of the rolled specimen under uniaxial compression in the transverse direction of rolling (2-2 axis). Where an isotropic specimen would show a positive strain in the lateral direction (1-1 axis), this sample shows almost zero strain, and actually negative. A similar anisotropic behavior of transformation strains is apparent on Figure 5-b as well. The point 2 corresponds to the response of the drawn specimen under equibiaxial tension. As opposed to the equal evolution of transformation strains for an isotropic material, in this case the material response favors the evolution of strains along the drawing direction. It appears that the effect of texture is much more prominent in the resulting transformation strains of the material than in yield stresses. The proposed evolution law seems able to approach the effect of processing to the end material behavior under proportional loading.

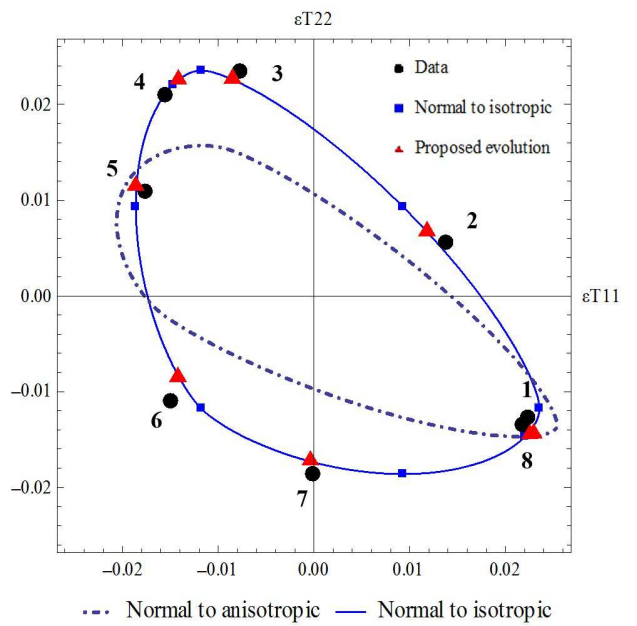

(a)

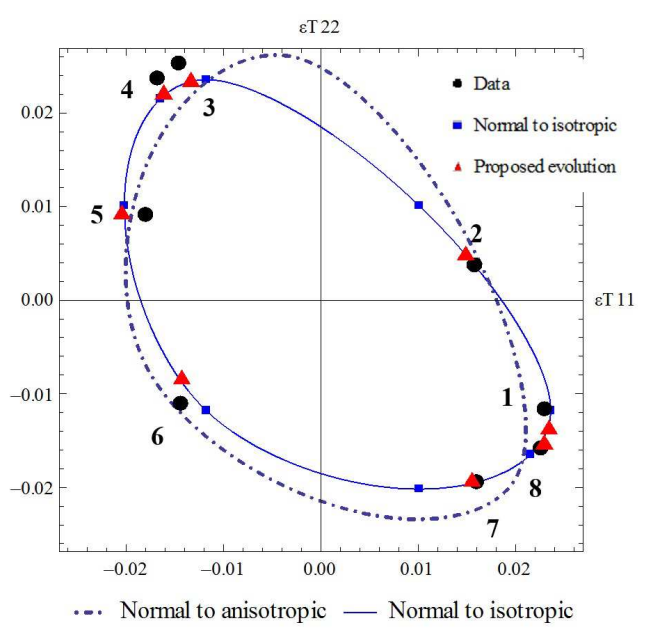

(b)

Figure 5: Projection of the resulting transformation strains according to the proposed evolution law for the rolled (a) and the drawn specimen (b) for $\xi=70 \%$ on the plane $\varepsilon_{\mathbf{1 1}}^{\boldsymbol{T}}-\varepsilon_{\mathbf{2 2}}^{\boldsymbol{T}}$. 


\section{Conclusion}

A new transformation criterion in terms of stresses and strains suitable for accurately describing the transformation of SMAs has been developed and implemented. The mathematical expressions governing the criterion in terms of stresses are studied with respect to convexity and capturing random anisotropy in SMAs transformation. Furthermore, an accurate evolution rule to govern the evolution of transformation strain has been formulated. It is a non-associated evolution rule which captures incompressibility and still the anisotropy in strains. The equations of the criterion and the evolution rule have been calibrated for a copper based textured SMA (Cu-Zn-Al), using the results from simulations of proportional uniaxial and biaxial plane-stress loading states. These simulations were achieved by utilizing the numerical results a self-consistent micromechanical model on three polycrystal configurations: isotropic, rolled and drawn. Further results of the model have been used to assess and establish the accuracy of the proposed anisotropic criterion and the related non-associated evolution rule. A good agreement has been obtained by comparing the micromechanical simulations to results provided by the new formulated macroscopic model that can be easily implemented in FE codes. Accordingly, the effects of asymmetry and anisotropy of SMAs behavior can be accounted for structural design of SMA actuators.

A key capability of the procedure used in this work to formulated and to calibrate the new transformation criterion and evolution equation is to establish a link between the processing conditions of a material and the final resulting macroscopic anisotropy of the overall behavior. Knowing the texture and the transformation parameters of a SMA polycrystal as a result of processing, and passing through the micromechanical model, the macroscopic behavior is simulated and then the anisotropic effect is captured without the requirement of a large experimental database of multiaxial loadings.

The use of this new criterion, combined with a thermodynamical model could extend the design capabilities of structures with highly textured SMAs. For such cases, the simulation of non-proportional loadings will be addressed in a future work.

\section{Acknowledgements}

The authors would like to acknowledge Drs. Darren Hartl and Björn Kiefer for motivating parts of the discussion that led this work to its final form, as well as for their support and input. The support of the NSF International Institute of Multifunctional Materials for Energy Conversion (IIMEC), award \#0841082, is acknowledged. The gratitude of the authors is also extended to Dr. Jean-Sébastien Lecomte for plotting the pole figures.

\section{References}

[1] C. Bouvet, S. Calloch, and C. Lexcellent, "A phenomenological model for pseudoelasticity of shape memory alloys under multiaxial proportional and nonproportional loadings," European Journal of Mechanics - A/Solids, vol. 23, pp. 37-61, Jan. 2004. 
[2] Y. Zhu and G. Dui, "A macro-constitutive model of polycrystalline niti \{SMAs\} including tensilecompressive asymmetry and torsion pseudoelastic behaviors," International Journal of Engineering Science, vol. 48, no. 12, pp. 2099 - 2106, 2010.

[3] E. Patoor, M. El Amrani, A. Eberhardt, and M. Berveiller, "Determination of the origin for the dissymmetry observed between tensile and compression tests on shape memory alloys," J. Phys., vol. IV, no. 2, pp. 495-500, 1995.

[4] P. Šittner and V. Novák, "Anisotropy of martensitic transformations in modeling of shape memory alloy polycrystals," International Journal of Plasticity, vol. 16, pp. 1243-1268, Jan. 2000.

[5] P. Sittner, L. Heller, J. Pilch, P. Sedlak, M. Frost, Y. Chemisky, A. Duval, B. Piotrowski, T. Ben Zineb, E. Patoor, F. Auricchio, S. Morganti, A. Reali, G. Rio, D. Favier, Y. Liu, E. Gibeau, C. Lexcellent, L. Boubakar, D. Hartl, S. Oehler, D. Lagoudas, and J. V. Humbeeck, "Roundrobin SMA modeling," in ESOMAT 2009 - 8th European Symposium on Martensitic Transformations, (Les Ulis, France), p. 08001, EDP Sciences, Sept. 2009.

[6] V. Grolleau, H. Louche, V. Delobelle, A. Penin, G. Rio, Y. Liu, and D. Favier, "Assessment of tensioncompression asymmetry of niti using circular bulge testing of thin plates," Scripta Materialia, vol. 65, no. 4, pp. 347-350, 2011.

[7] K. Taillard, S. A. Chirani, S. Calloch, and C. Lexcellent, "Equivalent transformation strain and its relation with martensite volume fraction for isotropic and anisotropic shape memory alloys," Mechanics of Materials, vol. 40, pp. 151-170, Apr. 2008.

[8] K. Taillard, Étude du comportement thermomécanique des alliages à mémoire de forme sous sollicitations multiaxiales complexes. Phd thesis, École Normale Supérieure de Cachan, 2006.

[9] E. Patoor, A. Eberhardt, and M. Berveiller, "Micromechanical Modelling of Superelasticity in Shape Memory Alloys," Journal de Physique IV, vol. 6, pp. C1-277, 1996.

[10] M. Somerday, R. J. Comstock, and J. A. Wert, "A systematic analysis of transformation stress anisotropy in shape memory alloys," Philosophical Magazine A, vol. 75, pp. 1193-1207, May 1997.

[11] C. Boehlert, S. Longanbach, and T. Bieler, "Effect of thermomechanical processing on the creep behaviour of Udimet alloy 188," Philosophical Magazine, vol. 88, pp. 641-664, Feb. 2008.

[12] D. Lagoudas, Shape Memory Alloys - Modeling and Engineering Applications. Springer, 2008.

[13] B. Coleman and M. Gurtin, "Thermodynamics with internal variables," J. Chem. Phys., vol. 47, no. 2, pp. 85-98, 1967. 
[14] B. Coleman and W. Noll, "The thermodynamics of elastic materials with heat conduction and viscocity," Arch. Rational Mech. Anal., vol. 13, p. 167, 1963.

[15] J. Lubliner, "On the thermodynamic foundations of non-linear solid mechanics," International Journal of Non-linear Mechanics, vol. 7, pp. 237-254, 1972.

[16] J. Lemaitre and J. L. Chaboche, Mechanics of Solid Materials. Cambridge University Press, 2002.

[17] J. Simo and T. Hughes, Computational Inelasticity. 1998.

[18] D. Lagoudas, D. Hartl, Y. Chemisky, L. Machado, and P. Popov, "Constitutive model for the numerical analysis of phase transformation in polycrystalline shape memory alloys," International Journal of Plasticity, vol. 32-33, pp. 155-183, May 2012.

[19] M. Qidwai and D. Lagoudas, "On thermomechanics and transformation surfaces of polycrystalline NiTi shape memory alloy material," International Journal of Plasticity, vol. 16, pp. 1309-1343, Jan. 2000.

[20] A. Karafillis and M. Boyce, "A general anisotropic yield criterion using bounds and a transformation weighting tensor," J. Mech. Phys. Solids, vol. 12, pp. 1859-1886, 1993.

[21] J. Boyd and D. Lagoudas, "A thermodynamical constitutive model for shape memory materials. Part I. The monolithic shape memory alloy," International Journal of Plasticity, vol. 12, pp. 805-842, Jan. 1996.

[22] D. J. Hartl, J. T. Mooney, D. C. Lagoudas, F. T. Calkins, and J. H. Mabe, "Use of a Ni60Ti shape memory alloy for active jet engine chevron application: II. Experimentally validated numerical analysis," Smart Materials and Structures, vol. 19, p. 015021, Jan. 2010.

[23] D. Entemeyer, Etude micromécanique du comportement thermomécanique des alliages à mémoire de forme. Phd, Université de Metz, 1996.

[24] S. Calloch, K. Taillard, S. Arbab Chirani, C. Lexcellent, and E. Patoor, "Relation between the martensite volume fraction and the equivalent transformation strain in shape memory alloys," Materials Science and Engineering: A, vol. 438-440, pp. 441444, Nov. 2006. 